Cite this: Chem. Commun., 2014, 50, 6704

Received 25th February 2014, Accepted 27th March 2014

DOI: $10.1039 / c 4 c c 01450 b$

www.rsc.org/chemcomm

\section{Molecular photo-oscillators based on highly accelerated heterocyclic azo dyes in nematic liquid crystals $\dagger$}

\author{
Jaume Garcia-Amorós, ${ }^{a}$ Marta Reig, ${ }^{a}$ M. Cidália R. Castro, ${ }^{b}$ Alba Cuadrado, ${ }^{a}$ \\ M. Manuela M. Raposo ${ }^{b}$ and Dolores Velasco*a
}

\begin{abstract}
Benzothiazole-pyrrole-based azo dyes greatly enhance their thermal isomerisation rate by up to 160 times when they are under the influence of the nematic mean field yielding the LC-based photochromic oscillators with the highest oscillation frequencies reported so far $(2.6 \mathrm{kHz}$ at $298 \mathrm{~K})$.
\end{abstract}

Liquid crystals (LCs) combine the molecular order of crystalline solids with the fluidity of conventional liquids. Particularly, nematic LCs are characterised by the tendency of their constituting molecules to point along a common direction. ${ }^{1}$ Doping of nematic LCs with light-sensitive molecules, like azo compounds, allows tuning the properties of the material in a quick and remote fashion by optical control. It is well known that azo chromophores exhibit a reversible photoisomerisation process between their trans (rod-like) and cis (bent-like) isomers; in addition, the metastable cis isomer also reverts back thermally in the dark. ${ }^{2}$ When azo-LC mixtures are illuminated, the bent cis-form of the azo chromophore is generated in the probe inducing changes in the organization at the molecular level. This effect is reflected in the variations of the properties of the material at the macroscopic scale, for instance, its ability to absorb light. ${ }^{3,4}$ This feature has given rise to many kinds of LC-based molecular photoswitchable materials. $^{5-8}$

Molecular photochromic oscillators modify their optical properties periodically and extremely fast upon exposure to light. Oscillating materials have attracted a great deal of attention over the last few years due to their huge applicability; e.g. artificial muscles for robotics, real-time optical information processors, rotary motors, switchable optical reflectors, etc. ${ }^{9-16}$

\footnotetext{
${ }^{a}$ Grup de Materials Orgànics, Institut de Nanociència i Nanotecnologia $\left(I N^{2} U B\right)$, Departament de Química Orgànica, Universitat de Barcelona, Martí i Franquès 1, E-08028, Barcelona, Spain. E-mail: dvelasco@ub.edu; Fax: +34 9333978 78; Tel: +34934039260

${ }^{b}$ Centro de Quimica, Universidade do Minho, Campus de Gualtar, 4710-057, Braga, Portugal

$\dagger$ Electronic supplementary information (ESI) available: Experimental part for the kinetic analyses and physical data for the LCs used. See DOI: 10.1039/c4cc01450b
}

For efficient photo-oscillation to be achieved, the azo dye used should undergo its thermal back conversion in a very quick fashion, most desirably within the microsecond timescale. Indeed, the overall performance of these materials relies on the control of the speed of such a process which, in turn, is mainly determined by the chemical architecture of the azo derivative. However, in LC-based materials, the azo dye must be introduced in an ordered host matrix to operate. In this medium, both the chemical nature and the dynamics of the host LC might influence the rate of the isomerisation process and, therefore, the oscillation frequency of the final material. Accordingly, achieving knowledge about how the host nematic matrix affects the rate of the thermal back conversion for very fast-isomerising azo dyes is of great importance for an optimal design of LC-based molecular photo-oscillators.

Positively-charged azo dyes have been recently described as the fastest molecular photochromic oscillators in neutral media since they exhibit thermal relaxation times as low as $33 \mu$ s at $298 \mathrm{~K}$ yielding, therefore, oscillation frequencies of up to $10 \mathrm{kHz} .{ }^{17}$ However, the low solubility of such azo chromophores in the most common host LC matrices limits their use as light-sensitive guests for LC-based materials. Thus, the formulation of new azo-LC mixtures, where the azo moiety shows not only a high solubility but also a very fast thermal isomerisation, still remains a challenge for achieving efficient LC-based photochromic oscillators.

Here we report on the role the nematic mean field plays on the thermal cis-to-trans isomerisation process for azo dyes that show relaxation times below the second timescale. We have found that benzothiazole-pyrrole-based azo compounds, which due to their neutral nature and rod-like geometry can be easily introduced as guests in a series of host LC matrices, enhance drastically their thermal isomerisation rate up to 160 times in these media. In fact, such azo derivatives exhibit thermal relaxation times at room temperature as low as $130 \mu \mathrm{s}$. This feature makes them the fastest non-positively-charged azo dyes in nematic LCs reported so far. In turn, these novel azo-LC mixtures enable high oscillation frequencies in the optical density of the material up to $2.6 \mathrm{kHz}$ under ambient conditions. 

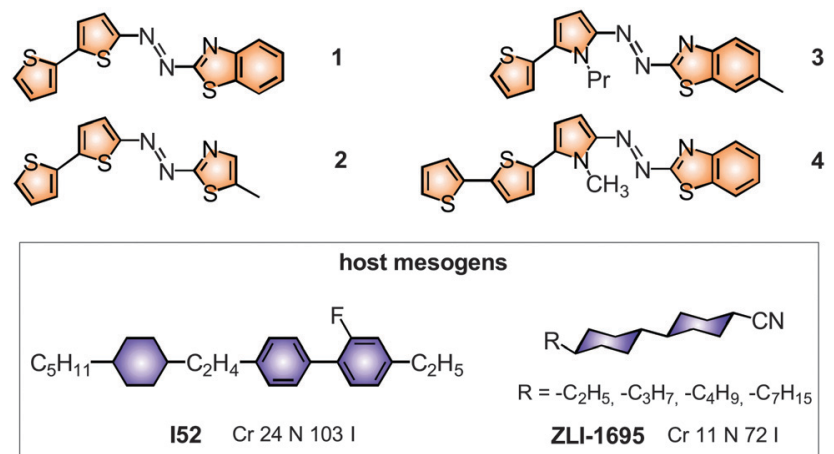

Scheme 1 Chemical structure of azo dyes 1-4 and nematic mesogens I52 and ZLI-1695. The phase transformation temperatures for the different LCs are given in Celsius.

Table 1 Relaxation time for the cis isomer and maximum oscillation frequencies for azo dyes 1-4 in different media at 298 K. $\tau$ and $\nu_{\max }$ are given in milliseconds and Hertz, respectively

\begin{tabular}{|c|c|c|c|c|c|c|c|c|c|c|}
\hline & \multicolumn{2}{|c|}{ Toluene } & \multicolumn{2}{|c|}{ Ethanol } & \multicolumn{2}{|c|}{ Acetonitrile } & \multicolumn{2}{|l|}{$\mathbf{I 5 2}$} & \multicolumn{2}{|c|}{ ZLI-1695 } \\
\hline & $\tau$ & $\nu_{\max }$ & $\tau$ & $\nu_{\max }$ & $\tau$ & $\nu_{\max }$ & $\tau$ & $\nu_{\max }$ & $\tau$ & $\nu_{\max }$ \\
\hline 1 & 64 & 5.2 & 108 & 3.1 & 65 & 5.1 & 170 & 2.0 & $-^{a}$ & $-^{a}$ \\
\hline 2 & 41 & 8.1 & 35 & 9.5 & 30 & 11 & 49 & 6.8 & $-^{a}$ & $-^{a}$ \\
\hline 3 & 20 & 17 & 1.0 & 330 & 5.6 & 60 & 1.4 & 240 & 17 & 20 \\
\hline 4 & 21 & 16 & 0.07 & 4830 & 0.44 & 760 & 0.13 & 2600 & 1.2 & 280 \\
\hline
\end{tabular}

The kinetics of the thermal cis-to-trans isomerisation for azo dyes 1-4 (Scheme 1) ${ }^{18}$ in both isotropic and nematic solutions were analysed by means of the nanosecond laser flash-photolysis technique (ESI $\dagger$ ). The thermal relaxation time of the cis isomers, $\tau(\tau=1 / k)$, was determined by fitting a monoexponential function to the data (Table 1). The maximum oscillation frequency of the corresponding photochromic oscillator was determined as $\nu_{\max }=1 /(3 \tau)$.

Whereas the relaxation times for the thermal cis-to-trans isomerisation process are very similar for compounds $\mathbf{1}$ and $\mathbf{2}$ in all the isotropic and nematic media analysed (Table 1), for pyrrole-containing azo compounds (3 and 4 ), clearly faster thermal back reactions are registered in both ethanol and acetonitrile than in toluene. Indeed, and despite the high polarity of acetonitrile, which is given by its dielectric permittivity ( $\varepsilon=37.5$ and 24.5 for acetonitrile and ethanol, respectively), the possibility of hydrogen bonding to be established in alcoholic solutions can induce an enhancement of the thermal isomerisation rate for such azo dyes. It is highly remarkable that the relaxation times found for compounds $\mathbf{3}$ and $\mathbf{4}$ in the nematic phase of mesogen $\mathbf{I 5 2}$ (Scheme 1) were of $1.4 \mathrm{~ms}$ and $130 \mu \mathrm{s}$, respectively (see Fig. 1 and 2a and Table 1), which are very close to the ones registered in ethanol solution, even though the polarities of both media are far from each other $(\varepsilon=2.91$ for I52).

A moderate increase of about 3-4 times in the thermal cis-totrans isomerisation rate has been described previously for other azo compounds when dissolved in a LC matrix. ${ }^{19-21}$ However, in this instance, the thermal isomerisation rate for azo compounds 3 and $\mathbf{4}$ in the nematic mesogen $\mathbf{I 5 2}$ was dramatically

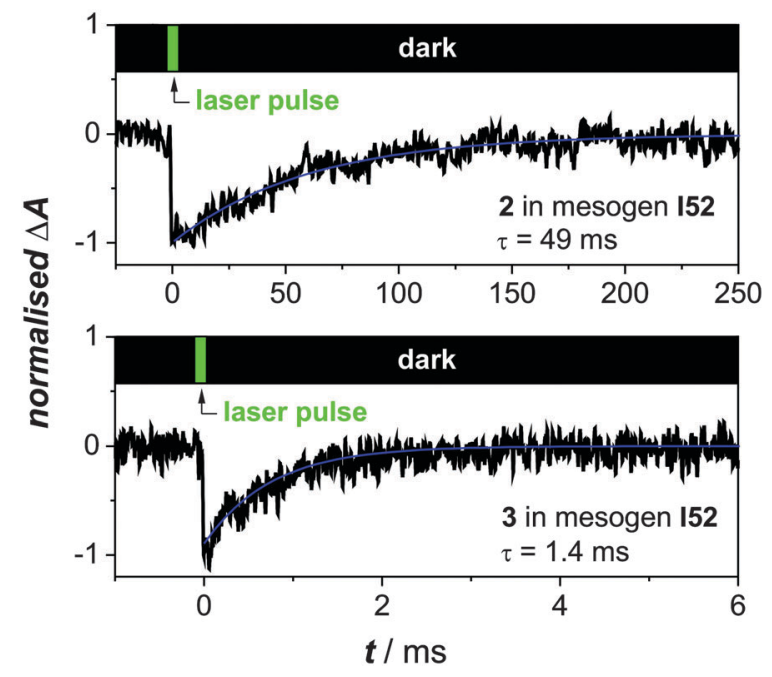

Fig. 1 Transient absorption change photoinduced by laser pulse irradiation $\left(\lambda_{\text {irrad }}=532 \mathrm{~nm}\right.$, Continuum Surelite $\mathrm{I}-10 \mathrm{Q}$-switched Nd-YAG laser with 5 ns pulse width and ca. $10 \mathrm{~mJ}$ per pulse) for azo dyes 2 and $\mathbf{3}$ in the mesogen 152 at $298 \mathrm{~K}\left(\lambda_{\text {obs }}=500 \mathrm{~nm},[\mathrm{AZO}]=4 \mathrm{mM}\right)$.

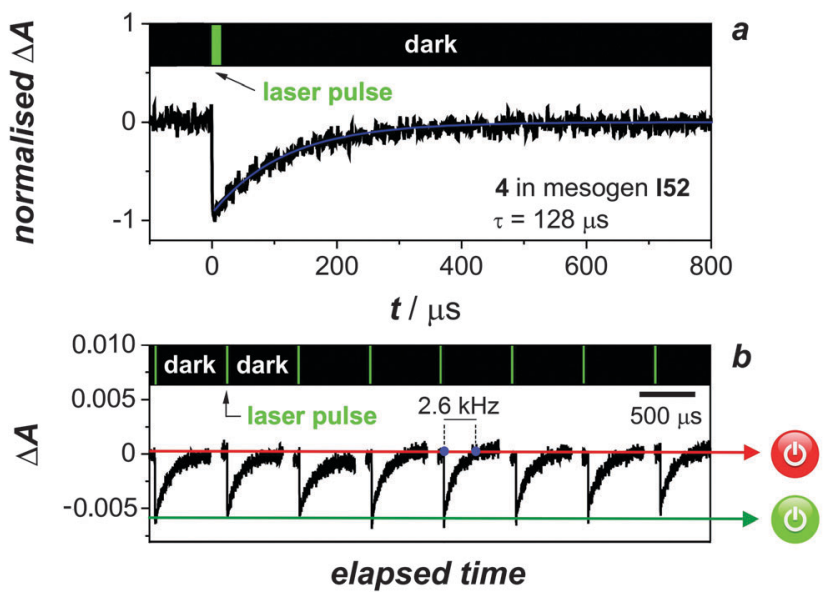

Fig. 2 (a) Transient absorption change photo-induced by laser pulse irradiation with green light $\left(\lambda_{\text {irrad }}=532 \mathrm{~nm}\right)$ at $298 \mathrm{~K}\left(\lambda_{\text {obs }}=500 \mathrm{~nm}\right)$ and (b) oscillation in the optical density of azo dye 4 in mesogen I52.

enhanced up to $c a .14$ and 160 times with respect to the corresponding ones in toluene, respectively, although these two media exhibit identical polarities ( $\varepsilon=2.38$ for toluene). Hence, the great enhancement of the thermal isomerisation rate observed for those azo compounds when dissolved in a nematic media should be clearly attributed mainly to the cooperative interaction between both azo dye and mesogen molecules that tend to recover their initial orientation along the nematic director once the illumination of the probe is stopped.

Previously reported systems thermally-isomerised via the inversion mechanism and, therefore, slow thermal back reactions were registered for them. ${ }^{19-21}$ However, for the azo dyes described herein the rotational pathway operates instead and, thus, the relaxation time of the azo dye falls within the very same timescale as that for the reorganization of the host LC 
towards the director direction. Indeed, it can be nicely seen from data displayed in Table 1 that the enhancement induced in the thermal back reaction by the host LC matrix decreases as the relaxation time of the azo dye increases, most probably because the relaxation time of the azo moiety becomes more different from the one for the LC reorientation.

In order to test the role the dynamics of the host LC matrix plays in the relaxation kinetics of the azo dye, the thermal back conversion for azo derivatives 3 and $\mathbf{4}$ was studied in the nematic phase of mesogen ZLI-1695 (Scheme 1). In this case, although the registered relaxation times fell within the same timescale as those found in mesogen $\mathbf{I 5 2}$ (17 and $1.2 \mathrm{~ms}$ for 3 and 4 in ZLI-1695, respectively, see Table 1), the enhancement produced by the nematic mean field was $c a$. 10-fold lower than the one detected in I52, pointing out that the dynamics of the host nematic mesophase can influence the thermal relaxation kinetics of the azo dye and, therefore, on the oscillation frequency of the final material. The dynamics of the host LC matrix is characterized by the viscoelastic coefficient $\left(K_{11} / \gamma_{1}\right),{ }^{22,23}$ where $K_{11}$ and $\gamma_{1}$ stand for the deformation elastic constant and the rotational viscosity, respectively. Mesogen 152 shows a $K_{11} / \gamma_{1}$ ratio of $5.21 \times 10^{-10} \mathrm{~m}^{2} \mathrm{~s}^{-1}$ at $298 \mathrm{~K}$, which is higher than the one exhibited by mesogen ZLI-1695 $\left(1.22 \times 10^{-10} \mathrm{~m}^{2} \mathrm{~s}^{-1}\right.$, see $\mathrm{ESI} \dagger$ for full numerical data). The shorter $K_{11} / \gamma_{1}$ value for mesogen ZLI-1695 is related to the longer time this mesogen needs to recover the initial orientation along the director after the illumination of the sample in comparison with mesogen $\mathbf{I 5 2}$. This feature stands for the higher enhancement that mesogen $\mathbf{I 5 2}$ induces on the azo dye thermal back conversion (see Table 1).

The very fast thermal isomerisation rate registered for the benzothiazole-pyrrole based azo dye 4 in the nematic mesogen I52 makes of this nematic mixture the best candidate to be applied as a light-controlled photochromic oscillator. Fig. $2 \mathrm{~b}$ shows the oscillation of the optical density of the $\mathbf{4}$ mixture with time upon continuous pulsed green light (532 nm)-dark cycles. The oscillation frequency of azo dye $\mathbf{4}$ in this nematogen is as high as $2.6 \mathrm{kHz}$ at room temperature (see Table 1) and, thus, a full cycle can be completed for that system in less than half of a millisecond. Moreover, the oscillating behaviour of such system remained unchanged after 12000 continuous working cycles. It should be highlighted that the oscillation frequency achieved with this system falls in the very same range as that reached in alcoholic solutions (up to $4.8 \mathrm{kHz}$ ) as well as those reported previously for positively-charged azobenzenes in isotropic solutions $(10 \mathrm{kHz})^{17}$ evidencing thereby the great potential applicability of the neutral azo-LC mixtures reported herein in photonic devices.

In summary, benzothiazole-pyrrole-based azo dyes enhance their thermal isomerisation rate up to 160 times when they are doped in low molar mass liquid crystals due to the influence of the nematic mean field. This enhancement has enabled the development of stable LC-based photochromic molecular oscillators with oscillation frequencies as high as $2.6 \mathrm{kHz}$ under ambient conditions for the first time.

Financial support for this research was obtained from the Ministerio de Ciencia e Innovación (Spain, CTQ2012-36074). J.G.-A. is grateful for a post-doctoral grant from the Generalitat de Catalunya (Spain, 2011 BP-A-00270). Thanks are also due to Fundação para a Ciência e Tecnologia (Portugal) for financial support to the portuguese NMR network (PTNMR, Bruker Avance III 400-Univ. Minho), FCT and FEDER (European Fund for Regional Development)-COMPETE-QREN-EU for financial support to the research centre CQ/UM PEst-C/QUI/UI0686/2013 (FCOMP-01-0124-FEDER-037302) and a PhD grant to M. C. R. C. (SFRH/BD/78037/2011).

\section{Notes and references}

1 G. W. Gray, Handbook of Liquid Crystals, Wiley-VCH, Weinheim, 1998.

2 H. Rau, Photochemistry and Photophysics, CRC Press, Boca Raton, Fl, 1990.

3 T. Ikeda, J. Mater. Chem., 2003, 13, 2037.

4 Y. Yu and T. Ikeda, J. Photochem. Photobiol., C, 2004, 5, 247.

5 X. Tong, M. Pelletier, A. Lasia and Y. Zhao, Angew. Chem., Int. Ed., 2008, 47, 3596.

6 Z. Alam, T. Yoshioka, T. Ogata, T. Nonaka and S. Kurihara, Chem. - Eur. J., 2007, 13, 2641.

7 J. Ma, Y. Li, T. White, A. Urbas and Q. Li, Chem. Commun., 2010, 46, 3463.

8 U. A. Hrozhyk, S. V. Serak, N. V. Tabiryan, L. Hoke, D. M. Steeves and B. R. Kimball, Opt. Express, 2010, 18, 8697.

9 S. Kobatake, S. Takami, H. Muto, T. Ishikawa and M. Irie, Nature, 2007, 446, 778.

10 T. J. White, N. V. Tabiryan, S. V. Serak, U. A. Hrozhyk, V. P. Tondiglia, H. Koerner, R. A. Vaia and T. J. Bunning, Soft Matter, 2008, 4, 1796.

11 M. Klok, N. Boyle, M. T. Pryce, A. Meetsma, W. R. Browne and B. L. Feringa, J. Am. Chem. Soc., 2008, 130, 10484.

12 C. L. Van Oosten, C. W. M. Bastiaansen and D. J. Broer, Nat. Mater., $2009,8,677$.

13 H. Koshima, N. Ojima and H. Uchimoto, J. Am. Chem. Soc., 2009, 131, 6890.

14 J. Horvath, I. Szalai, J. Boissonade and P. De Kepper, Soft Matter, 2011, 7, 8462.

15 K. M. Lee and T. J. White, Polymers, 2011, 3, 1447.

16 U. A. Hrozhyk, S. V. Serak, N. V. Tabiryan, T. J. White and T. J. Bunning, Opt. Mater. Express, 2011, 1, 943.

17 J. Garcia-Amorós, S. Nonell and D. Velasco, Chem. Commun., 2011, 47, 4022.

18 J. Garcia-Amorós, M. Cidália, R. Castro, P. Coelho, M. Manuela, M. Raposo and D. Velasco, Chem. Commun., 2013, 49, 11427.

19 J. Garcia-Amorós, M. Martínez, H. Finkelmann and D. Velasco, J. Phys. Chem. B, 2010, 114, 1287.

20 J. Garcia-Amorós, H. Finkelmann and D. Velasco, Phys. Chem. Chem. Phys., 2011, 13, 11233.

21 J. Garcia-Amorós, H. Finkelmann and D. Velasco, Chem. - Eur. J., 2011, 17, 6518.

22 S.-T. Wu, M. E. Neubert, S. S. Keast, D. G. Abdallah, S. N. Lee, M. E. Walsh and T. A. Dorschner, Appl. Phys. Lett., 2000, 77, 957.

23 S. Gauza, H. Wang, C.-H. Wen, S.-T. Wu, A. J. Seed and R. Dabrowski, Jpn. J. Appl. Phys., 2003, 42, 3463. 